\title{
Competing Roles of longitudinal and transverse Hund's terms on Mott transitions
}

\author{
Ya-Min Quan ${ }^{1}$,Xiang-Long $\mathrm{Yu}^{1}$,Qing-Wei Wang ${ }^{1}$ and Liang-Jian Zou ${ }^{1,2}$ * \\ ${ }^{1}$ Key Laboratory of Materials Physics, \\ Institute of Solid State Physics, \\ Chinese Academy of Sciences, \\ Hefei 230031, P.R. China \\ ${ }^{2}$ University of Science and Technology of China,
}

Hefei 230026, China

(Dated: today)

\begin{abstract}
Effects of longitudinal $\left(J_{Z}\right)$ and transverse (including spin-flip $J_{X}$ and pair-hopping $J_{P}$ terms) Hund's couplings on Mott transitions of two-orbital Hubbard models are studied by the rotationally invariant salve boson approach. We show that in the half-filled asymmetric systems, the orbital selective Mott phase (OSMP) expands with increasing $J_{X, P} / J_{Z}$ when $J_{X, P} / J_{Z}<1$, and has the largest region in the isotropic case $\left(J_{X, P} / J_{Z}=1\right)$; and further increasing spin-flip Hund's coupling to $J_{X, P} / J_{Z}>1$ may quickly suppress the OSMP state. In other near-half-filled systems, the transverse Hund's coupling favors or unfavors the OSMP state, depending on the electronic correlation strength of the systems. In the quarter-filled and around systems, a small $J_{X, P} / J_{Z}<1$ has less effect on Mott transition, while a large $J_{X, P} / J_{Z}>1$ enhances the electron itineracy and considerably increases the critical correlation strength of the Mott transition both in symmetric and asymmetric systems. These results could be addressed by different spin-orbital states favored by $J_{X}, J_{P}$ and $J_{Z}$ components, respectively; and the competing longitudinal and transverse Hund's coupling terms lead to most strong quantum fluctuations in the isotropic system.
\end{abstract}

PACS numbers: $71.30 .+\mathrm{h}, 71.10 . \mathrm{Fd}, 71.27 .+\mathrm{a}$

\footnotetext{
* Correspondence author, Electronic mail:zou@theory.issp.ac.cn
} 


\section{INTRODUCTION}

In Mott metal-insulator transition (MIT) in multi-orbital systems, strong Coulomb interaction may first drive an orbital to insulating, and the others remain metallic, forming the so-called orbital selective Mott phase (OSMP) [1]. This phase has received great attention in the past years [2-11], recently it was even related to iron-based superconductors. The possible relationship between the OSMP and the bad metallicity and the coexistence of itinerant and localized electrons in parent phases of some iron-pnictide and iron-chalcogenide superconductors have attracted great interests both experimentally and theoretically [12-25]. For example, Rong et al. showed that with increasing electron correlation, the electrons in degenerate $d_{x z / y z}$ orbitals remain itinerant and the quasi-particle weight of the $d_{x y}$ orbital becomes zero[21], suggesting the existence of OSMP in $\mathrm{A}_{x} \mathrm{Fe}_{2-y} \mathrm{Se}_{2}(\mathrm{~A}=\mathrm{K}, \mathrm{Rb})$; on the other hand, it was found that the electron doping significantly modifies the electron correlations, particle distributions and quasi-particle weights of the $3 \mathrm{~d}$ orbitals, hence seriously influences the OSMP in iron-based compounds [24, 25]. Besides the Coulomb correlation and the doping effect, the OSMP may also be driven by asymmetric bandwidths, crystal field splitting and Hund's coupling [9, 23, 26-28]. Among these factors, the Hund's coupling plays a distinct role in Mott transitions in ironpnictides and other multiorbital correlated systems. Due to considerable role of Hund's coupling on the low-energy processes, the normal phase of iron-based superconductors and other multiorbital correlated systems are called as Hund's metal $[20,24,29,30]$. The essential properties of Hund's metals receives considerable attention these years $[7,10,31]$.

In a half-filled two-orbital system without the Hund's coupling, with increasing correlation, the system undergoes a Mott transition from a paramagnetic (PM) metallic phase into a low-spin band insulator or orbital-Mott phase $[5,22,32]$. The two orbitals become insulating simultaneously. Once an Ising-type Hund's coupling term is turned on, the effective correlation is enhanced and the critical correlation strength for MIT considerably reduces in degenerate systems; in the nondegenerate two-orbital Hubbard systems, an OSMP appears in a very small region [8,33-35] in the PM phase diagram. Further, when the isotropic Hund's coupling is considered in the nondegenerate two-orbital system, the transverse Hund's coupling term enlarges the OSMP regime in the phase diagram $[5,7,10]$ and the first-order character of MIT becomes weak [36], in comparison with the the Ising-type cases. These results demonstrate that the longitudinal and transverse components of the Hund's coupling play interesting and complex roles in MIT and OSMP through 
modulating the effective electron correlations and the spin and orbital fluctuations. In realistic compounds, due to the crystalline field anisotropy and magnetic crystalline anisotropy, the conventional rotational invariance $\left(J_{X, P}=J_{Z}\right)$ in free space is usually broken in realistic anisotropic systems, so it is necessary to disclose the effects of $J_{X, P} \neq J_{Z}$. Nevertheless, the effective roles of the Ising-type longitudinal component and the spin-flip and pair-hopping transverse component in MIT and OSMT are far from well understood yet.

It is essential to distinguish the contributions of the longitudinal and transverse components of the Hund's coupling on MIT and OSMT. In comparison with the well-established results with $J_{X, P}=0$, turning on $J_{X, P}$ gradually could uncover the competing role between $J_{X, P}$ and $J_{Z}$ on the ground state of strongly correlated systems. This overcomes the shortage of a posteriori of the usual dropping of $J_{X, P}$ for practicality in previously numerical calculations. In addition, the gradual increase of the transverse components $J_{X, P}$ could mimic the enhancement of spin/orbital fluctuations in Hund's metals and other strongly correlated systems, which are helpful to understand the underlying low-energy physics in multi-orbital systems, especially for the parent phases of mutliorbital ironpnictide superconductors, which are regarded as Hund's metals [20, 24, 29, 30]. In this paper, we mainly focus on anisotropic Hund's rule coupling and study the competing roles of the longitudinal and transverse Hund's coupling terms on spin and orbital fluctuations and OSMP in two-orbital Hubbard models. We show that the transverse Hund's terms could seriously affect the electron correlation of the systems and Mott transitions. This paper is organized as follows: we firstly describe the model Hamiltonian and theoretical approach in Sec.II, then in Sec. III we present the effects of different part of Hund's rule coupling, and orbital degree of freedom on MIT and OSMP are discussed. The final section is devoted to the concluding remarks.

\section{MODEL HAMILTONIAN AND METHODS}

We start with the degenerate Multi-orbital Hubbard model with rectangular density of states (DOS). We choose rectangular DOS for the reason that the results do not qualitatively depend on the exact choice of the bare DOS for saving much computational time. The Hamiltonian is given 


$$
\begin{aligned}
H= & H_{k i n}+H_{l o c} \\
H_{k i n}= & \sum_{<i j>, \alpha, \beta, \sigma}\left(t_{i j}^{\alpha, \beta} c_{i \alpha \sigma}^{\dagger} c_{j \beta, \sigma}+\text { h.c. }\right)+\sum_{i, \alpha, \sigma}\left(\varepsilon_{\alpha}-\mu\right) n_{i \alpha \sigma} \\
H_{l o c}= & U \sum_{i, \alpha} n_{i \alpha \uparrow} n_{i \alpha \downarrow}+\sum_{i, \sigma, \sigma^{\prime}, \alpha>\beta}\left(U^{\prime}-J_{Z} \delta_{\sigma \sigma^{\prime}}\right) n_{i \alpha \sigma} n_{i \beta \sigma^{\prime}} \\
& -J_{X} \sum_{i, \alpha \neq \beta}\left(c_{i \alpha \uparrow}^{\dagger} c_{i \alpha \downarrow} c_{i \beta \downarrow}^{\dagger} c_{i \beta \uparrow}\right)+J_{P} \sum_{i, \alpha \neq \beta}\left(c_{i \alpha \uparrow}^{\dagger} c_{i \alpha \downarrow}^{\dagger} c_{i \beta \downarrow} c_{i \beta \uparrow}\right)
\end{aligned}
$$

where $c_{i \alpha \sigma}^{\dagger}$ creates an electron with the orbital index $\alpha$ and spin $\sigma$ at the lattice site $i, \varepsilon_{\alpha}$ is the energy level of the $\alpha$ orbital, $n_{i \alpha \sigma}$ is the corresponding occupation number operator. $t_{i j}^{\alpha, \beta}$ is the hopping integral between two orbitals $\alpha$ and $\beta$. The intra-orbital (inter-orbital) Coulomb repulsion is $U\left(U^{\prime}\right)$. The Hund's rule coupling of the density-density interaction, spin-flip and pair-hopping terms are denoted as $J_{Z}, J_{X}$ and $J_{P}$, respectively. Throughout this paper we set $U^{\prime}=U-2 J_{Z}$, and $J_{Z}=U / 4$, unless otherwise specified.

The slave boson theory was firstly introduced by Kotliar and Ruckenstein [37, 38]. In this slave boson theory framework, the density-density interaction are expressed as a function of slave boson fields. The ground states of the system is found by optimizing the saddle approximation values of the slave bosons. This method is suitable for dealing with arbitrary interaction strengths and complex magnetic structures[39, 40]. To treat with arbitrary form of interacting Hamiltonian and noncollinear magnetic configuration, the Kotliar-Ruckenstein slave boson theory was extended to rotationally invariant slave boson (RISB) theory [36, 41, 42], which is more convenient in studying the effects of the full Hund's coupling on MIT and OSMP. It is especially useful for distinguishing the different roles of the transverse and longitudinal components of the Hund's coupling.

To find the ground state of the two-orbital Hubbard model with fully Hund's coupling terms, we project the original Hamiltonian in Eq. (1) into the RISB representation [36]. In the RISB framework, the electron creation operator is represented in terms of bosons and quasiparticle operators as:

$$
c_{\xi}^{\dagger}=\sum_{\eta} \hat{R}_{\xi \eta}^{*} f_{\eta}^{\dagger}
$$

where $f_{\eta}^{\dagger}$ is a auxiliary fermion operator and $\hat{R}_{\xi \eta}^{*}$ is the renormalization matrix. In the RISB expression, the combined spin-orbital indexes $\xi$ and $\eta$ label the quantum states of the electrons and 
quasiparticles, which include the spin and orbital for convenience. The renormalization matrix is defined as

$$
\hat{R}_{\xi \eta}^{*}=\sum_{A B n m \gamma} \widehat{C}_{B m}^{A n}(\xi, \gamma) \phi_{A n}^{\dagger} \phi_{B m} M_{\gamma \eta}
$$

with

$$
\widehat{C}_{B m}^{A n}(\xi, \gamma)=\left\langle A\left|c_{\xi}^{\dagger}\right| B\right\rangle\left\langle n\left|f_{\gamma}^{\dagger}\right| m\right\rangle
$$

Here $\phi_{A n}^{\dagger}$ and $\phi_{B m}$ are the slave boson operators[36]. The first indexes $A$ and $B$ refer to the physical electron Fock states $|A\rangle$ and $|B\rangle$. The second indexes $n$ and $m$ refer to the quasiparticle Fock states $|n\rangle$ and $|m\rangle$. The normalization coefficient matrix $M_{\gamma \eta}$ is used to ensure the renormalization factors be unity for noninteracting case. The expression of $M_{\gamma \eta}$ reads

$$
M_{\gamma \eta} \equiv\left\langle\gamma\left|\left[\frac{1}{2}\left(\hat{\Delta}^{(p)} \hat{\Delta}^{(h)}+\hat{\Delta}^{(h)} \hat{\Delta}^{(p)}\right)\right]^{-1 / 2}\right| \eta\right\rangle .
$$

One notices that in the collinear case, such as paramagnetic, Ferromagnetic and Néel antiferromagnetic ground states, only the diagonal elements of matrixes $\hat{R}_{\xi \eta}^{*}$ and $M_{\gamma \eta}$ play active roles. The matrix of the QP weight is obtained in terms of the $\hat{R}_{\xi \eta}$ matrix at the saddle point as $\mathbf{q}=\mathbf{R}^{\dagger}$. The expressions of quasiparticle and quasihole density matrices $\hat{\Delta}^{(p)}$ and $\hat{\Delta}^{(h)}$ in Eq.(7) are given by

$$
\begin{aligned}
& \hat{\Delta}_{\xi \eta}^{(p)}[\phi] \equiv \sum_{A m n} \phi_{A n}^{*} \phi_{A m}\left\langle m\left|f_{\xi}^{\dagger} f_{\eta}\right| n\right\rangle \\
& \hat{\Delta}_{\xi \eta}^{(h)}[\phi] \equiv \sum_{A m n} \phi_{A n}^{*} \phi_{A m}\left\langle m\left|f_{\eta} f_{\xi}^{\dagger}\right| n\right\rangle .
\end{aligned}
$$

Therefore the original Hamiltonian can be written in terms of the slave bosons. In the mean field approach, the projected Hamiltonian is expressed in terms of the slave bosons as

$$
\begin{aligned}
H= & \sum_{<i j>, \xi, \xi^{\prime}, \eta, \eta^{\prime}}\left(t_{i j}^{\xi, \eta} \hat{R}_{\xi \xi^{\prime}}^{*} \hat{R}_{\eta \eta^{\prime}} f_{i \xi^{\prime}}^{\dagger} f_{i \eta^{\prime}}+\text { h.c. }\right)+\sum_{i, \xi}\left(\varepsilon_{\xi}-\mu\right) f_{i \xi}^{\dagger} f_{i \xi} \\
& +\sum_{A B}\left\langle A\left|H_{l o c}\right| B\right\rangle \sum_{n} \phi_{A n}^{\dagger} \phi_{B n},
\end{aligned}
$$

To ensure the solution in physical Hilbert subspace, we should adopt the normalization constraint and fermion number constraints defined as follows[36]:

$$
\sum_{A n} \phi_{A n}^{\dagger} \phi_{A n}=1
$$


and

$$
\sum_{A} \sum_{n m} \phi_{A m}^{\dagger} \phi_{A n}\left\langle n\left|f_{\xi}^{\dagger} f_{\eta}\right| m\right\rangle=f_{\xi}^{\dagger} f_{\eta}, \quad \forall \xi \eta
$$

We obtain the ground state by solving the self-consistent equations through minimizing the average of Eq. (10) in the saddle-point approximation [43,44]. Throughout this paper we use the rectangular DOS for the two orbitals, and set $D_{1}$ and $D_{2}$ as the half bandwidths of orbitals 1 and 2 , respectively. We will mainly focus on the different influences of the spin-flip and pair-hopping terms and the Ising-type term on MIT and OSMP.

\section{NUMERICAL RESULTS}

As stated above, longitudinal and transverse components of the Hund's coupling play competing roles[6, 7]. In order to clarify the roles of the longitudinal and transverse components in MIT and OSMP, we study the dependence of the QP weights and correlation functions on Coulomb correlation and anisotropic Hund's coupling for quarter-filling $(n=1)$ and half-filling $(n=2)$ cases, as well as away from these cases.

\section{A. Quarter-filling case}

We first present the correlation-anisotropy phase diagram of the quasiparticle $(\mathrm{QP})$ weight in degenerate two-orbital systems in Fig.1. In the blue region, the QP weights of the system are zero, indicating two orbits are localized and the system is a Mott state in the strongly correlation regime; in the green region, the QP weight is finite and considerably small than unity, indicating a strongly correlated bad metal; and in the red region, the QP weight is intermediate finite, the bandwidths of the system are weak or moderate renormalized, indicating that the ground state of the system is a good metal. Obviously, the critical correlation strength $U_{c}$ for MIT first slightly increases with increasing transverse Hund's terms when $J_{X, P} / J_{Z}<1$. This is attributed to the fact that the interorbital charge and spin fluctuations are small at $J_{X, P} / J_{Z}<1$. In the region of large transverse Hund's coupling with $J_{X, P} / J_{Z}>1, U_{c}$ steeply raises up with the lift of $J_{X, P} / J_{Z}$, as seen in Fig.1. The increase of transverse component significantly enhances the interorbital charge and spin fluctuations, and lifts the QP weights and the electron itineracy, leading to the considerable increase of $U_{c}$. It is reasonably expected that at quarter filling, increasing Ising-type Hund's term 


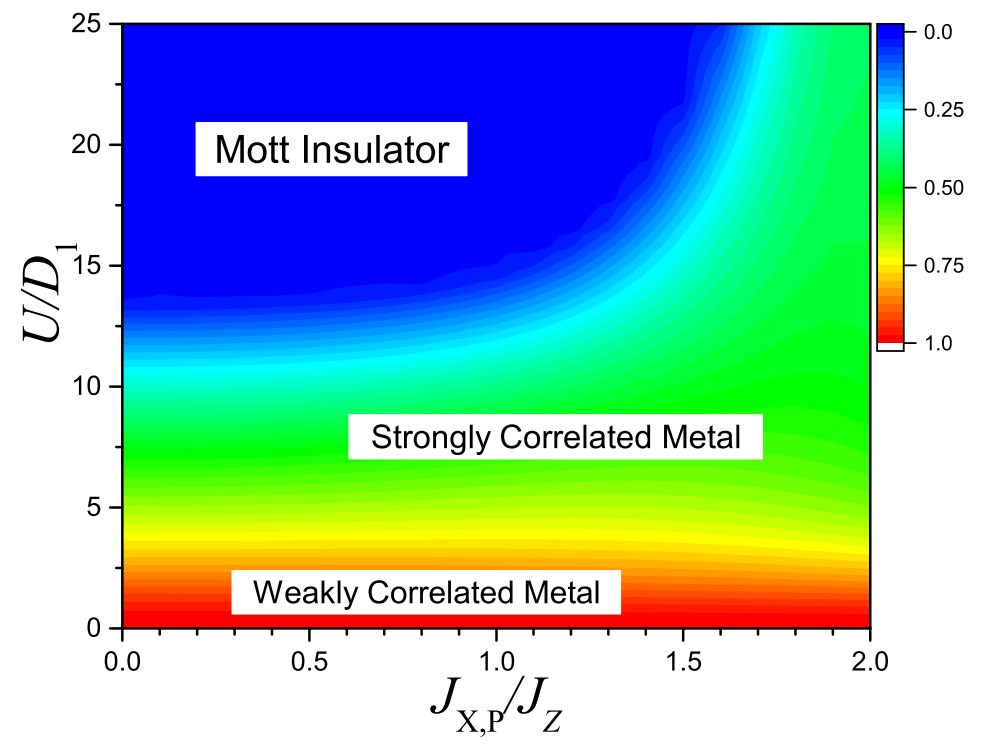

FIG. 1: The quasiparticle-weight phase diagram of electron correlation $U / D_{1} v s$ anisotropic Hund's rule coupling $J_{X, P} / J_{Z}$ at quarter filling. Red and green regions are metallic phase and blue region is insulating phase. Theoretical parameters are $J_{Z}=0.25 U$ and $D_{2}=D_{1}$.

also leads to the lift of $U_{c}$, as observed in [30], however, we find that the magnetic ground states of the systems with large $J_{X, P}$ and with $\operatorname{large} J_{Z}$ are different. In the nondegenerate case with $D_{2}=2 D_{1}$, we obtain similar results to Fig. 1 .

To further reveal the mechanism of MIT in the presence of transverse Hund's coupling, we plot the interorbital charge and spin correlation functions as the functions of $J_{X, P} / J_{Z}$ in Fig.2. These interorbital correlations and fluctuations as the functions of electron correlation $U / D_{1}$ are also plotted in the insets in Fig.2. From Fig.2(a), we find that only when the transverse Hund's terms become large to $J_{X, P} / J_{Z}>1 \sim 1.5$, the interorbital charge correlations are considerably affected by the transverse Hund's terms. Since the electrons tend to distribute in different orbitals when $J_{X, P} / J_{Z}<1$. As large spin-flip and pair-hopping Hund's term are turned on, the interorbital charge fluctuation is suppressed, and it approaches to zero when spin-flip and pair-hopping terms $J_{X, P} / J_{Z}$ become large. At $J_{X, P} / J_{Z}=2$, the interorbital charge correlation function becomes closing to zero in the strong correlation regime. The MIT is also not found in the quarter filling case, as shown in the inset of Fig.2(a). In contrast, the interorbital spin correlation are enhanced paramagnetically or ferromagnetically for small $J_{X, P} / J_{Z}$, or antiferromagnetically for large $J_{X, P} / J_{Z}$, as shown in Fig.2(b). This is due to the fact that when $J_{X, P} / J_{Z}<1$, the z-components of electron spins in different orbitals are parallel; when $J_{X, P} / J_{Z}>1$, large enough $J_{X, P}$ makes the electron spins tend 

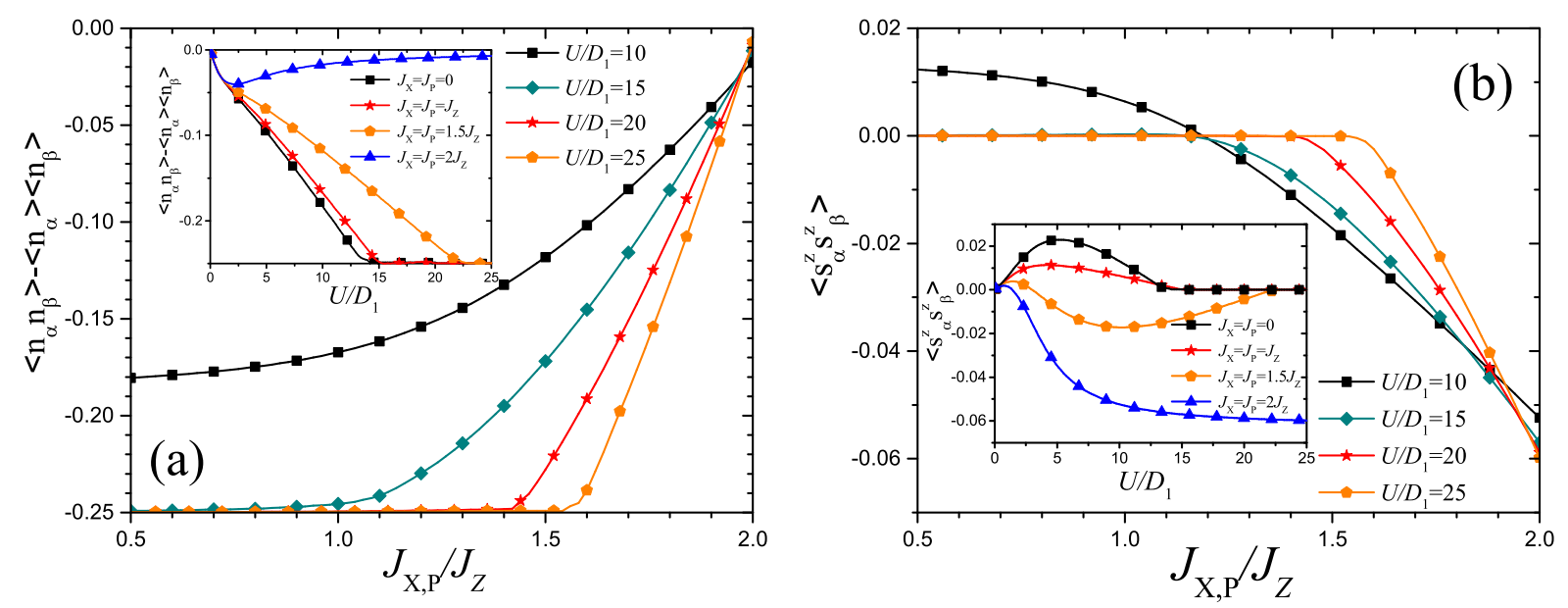

FIG. 2: The interorbital charge correlations (a) and spin correlation functions (b) as a function of transverse Hund's term for different electron correlations with $D_{2}=D_{1}$ and $J_{X}=J_{P}$ at $n=1$. The insets in (a) and (b) display these correlation functions as a function of $U / D_{1}$ at $J_{X, P}=0$ (black square), $J_{Z}$ (red star), $1.5 J_{Z}$ (orange pentagon), and $2 J_{Z}$ (blue up triangle), respectively..

to antiparallel in z direction. Such a complicated behavior is also seen in the correlation-dependent interorbital spin correlation functions, as shown in the inset of Fig.2(b). In a quarter-filled system, the weight of double occupation on the lattice sites is very small, and the single occupation is dominant. Thus the effects of the pair-hopping Hund's terms on the intraorbital charge fluctuations and spin moments are negligible.

\section{B. Half-filling case}

Different from the quarter-filing case, the interorbital spin and charge correlations and intraorbital charge and spin fluctuations all seriously depend on the transverse Hund's terms. We plot the orbital-resolved QP weights of half-filled systems with $D_{2}=2 D_{1}$ in Fig. 3 (a) for orbital-1 and Fig. 3 (b) for orbital-2. It shows that the system is a weakly correlated metal when $U / D_{1}<2$ and a strongly correlated metal when $U / D_{1}<4$, as one expects. In the between, electron correlation drives narrow orbital-1 into insulating and wide orbital-2 remains metallic, giving rise to OSMP phase, as indicated in the white dash-line region in Fig. 3. In this case the transverse Hund's coupling acts distinct roles on the MIT of narrow and wide bands. As seen in Fig. 3(a), the critical electron correlation of the narrow orbital for MIT monotonically decreases with increasing $J_{X, P}$. On the contrary, the critical electron correlation of wide orbital-2 increases firstly and then de- 

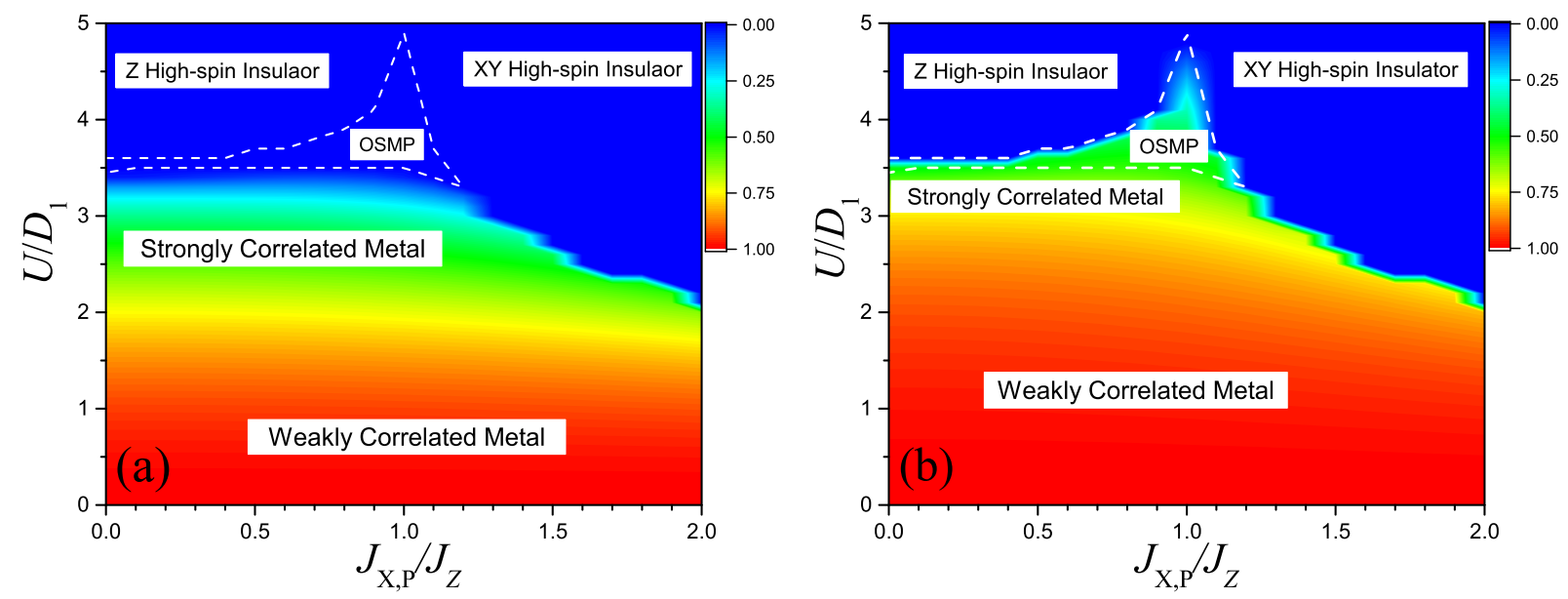

FIG. 3: The quasiparticle weights of orbital 1 (a) and orbital 2 (b) vs correlation $U / D_{1}$ and transverse Hund's rule coupling at $n=2$. White dashed lines denote the OSMP region. The bandwidth parameter is $D_{2}=2 D_{1}$.

creases with increasing $J_{X, P}$, and the OSMP region separates the high-spin insulating phase from the low-spin one, implying that increasing spin-flip Hund's coupling term drives the high-low spin state transition. Due to different influences of the transverse Hund's rule coupling on these two orbitals, the OSMP is mostly favored by the $J_{X, P}$ in the vicinity of $J_{X, P} / J_{Z}=1$. With $J_{X, P} / J_{Z}>1$, the OSMP region is greatly suppressed. Thus, the isotropic system with $J_{X, P}=J_{Z}$ is a special case, where the OSMP can exist over a wide electron correlation range, and the MIT is nearly a continuous phase transition; while the MIT in anisotropic systems remain the first order.

The effects of the transverse Hund's terms on charge and spin fluctuations in three typical correlated systems $\left(U / D_{1}=2,3\right.$, and 4) are shown in Fig. 4. As shown in Fig.4(a), at $U / D_{1}=2$, the interorbital charge correlation function increases with lifting of $J_{X, P} / J_{Z}$; while it decreases with increasing spin-flip Hund's term at $U / D_{1}=3$; in the insulating regime, the interorbital charge fluctuation decreases to zero, implying the decoupling of charge degree of freedom of two orbitals, in agreement with de Medici et al.'s results [10]. The inset of Fig.4(a) shows that the interorbital charge correlation function is the most strongest in the intermediate correlation regime, and the correlation-driven MIT is the first order when $J_{X, P} / J_{Z}=1.5$ and 2.

In a contrast to the decoupling of charge degree of freedom between two orbitals in the insulating regime, the coupling of the spin degree of freedom of two orbitals are strong, as one sees in the dependence of interorbital spin correlation functions on transverse Hund's rule coupling terms in 

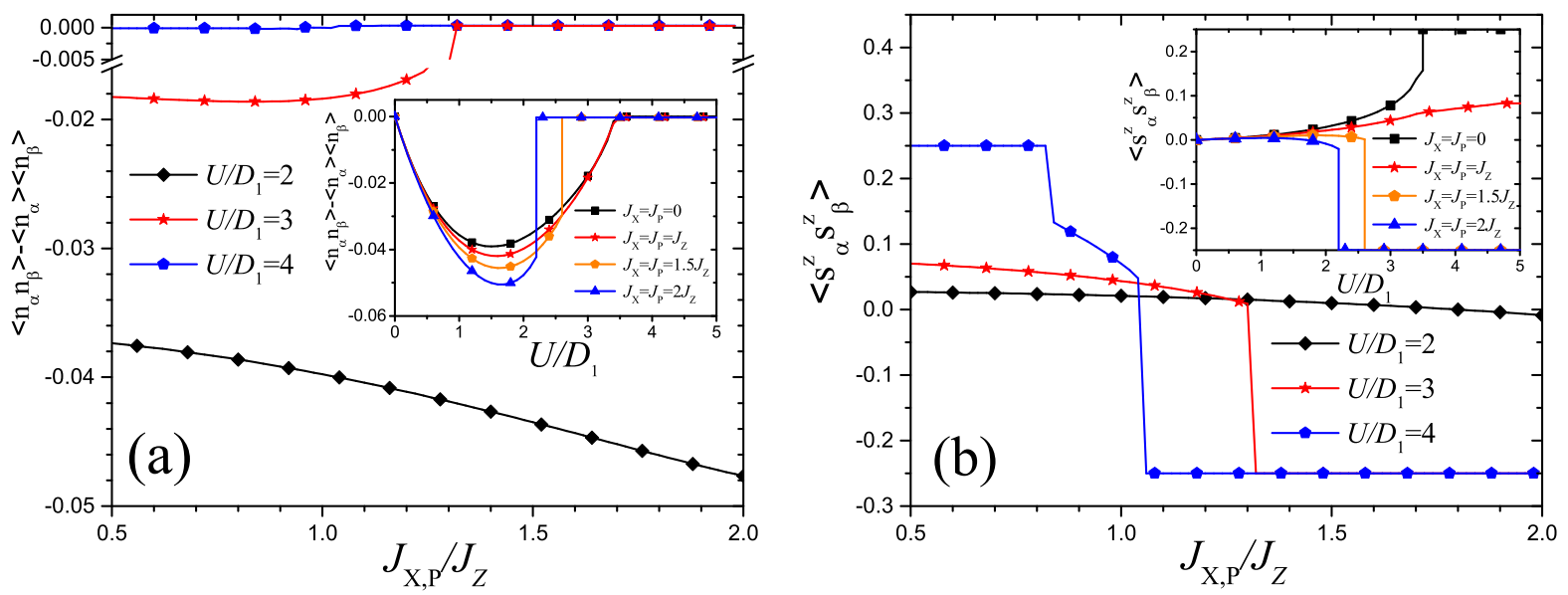

FIG. 4: The interorbital charge correlation functions (a) and z-component correlation functions of spins (b) as the functions of transverse Hund's term at $D_{2}=2 D_{1}$ and $n=2$ for different electron correlations. The insets in (a) and (b) show the results as a function of $U / D_{1}$ at $J_{X, P} / J_{Z}=0$ (black square), 1 (red star), 1.5 (orange pentagon), and 2 (blue up triangle), respectively.

Fig.4(b). The interorbital spin correlation functions $\left\langle S_{\alpha}^{Z} S_{\beta}^{Z}>\right.$ are antiferromagnetic and approach to -0.25 in the Mott insulating phase for $J_{X, P} / J_{Z}>1.5$, and are ferromagnetic when $J_{X, P} / J_{Z}<1$. Especially, the z-component correlation $<S_{\alpha}^{Z} S_{\beta}^{Z}>$ demonstrates a complicated $J_{X, P}$ dependence in the OSMP regime, indicating an intermediate spin state. The Inset of Fig.4(b) displays the $U$ dependence of the interorbital spin correlations for different $J_{X, P}$, in consistent with those of Fig .4(b)

From these results, we arrive a clear scenario on the effect of the transverse Hund's coupling on two-orbital MIT/OSMT: the OSMP phase is not only a partially localization charge state, but also an intermediate spin state with strong spin fluctuations. In this phase, the total spin is contributed by the local and itinerant electrons coupled by the exchange interaction[45]. As shown in the preceding, the OSMP state separates two high-spin insulating states, it is a strongly spin-fluctuating system.

Furthermore, we plot the moment square of total spin $\left\langle\vec{S}^{2}\right\rangle$ and the moment square of spins in each orbital $\left\langle\vec{S}_{\alpha}^{2}>\right.$ in Fig.5. As shown in Fig.5(a), the moment square of total spin increases with lifting transverse Hund's terms in the intermediate correlation region. In the strongly correlated regime, the moment square of total spin becomes largest and is independent of the transverse Hund's terms both for $J_{X, P} / J_{Z}<1$ and for $J_{X, P} / J_{Z}>1$. Particularly, the OSMP region appears 

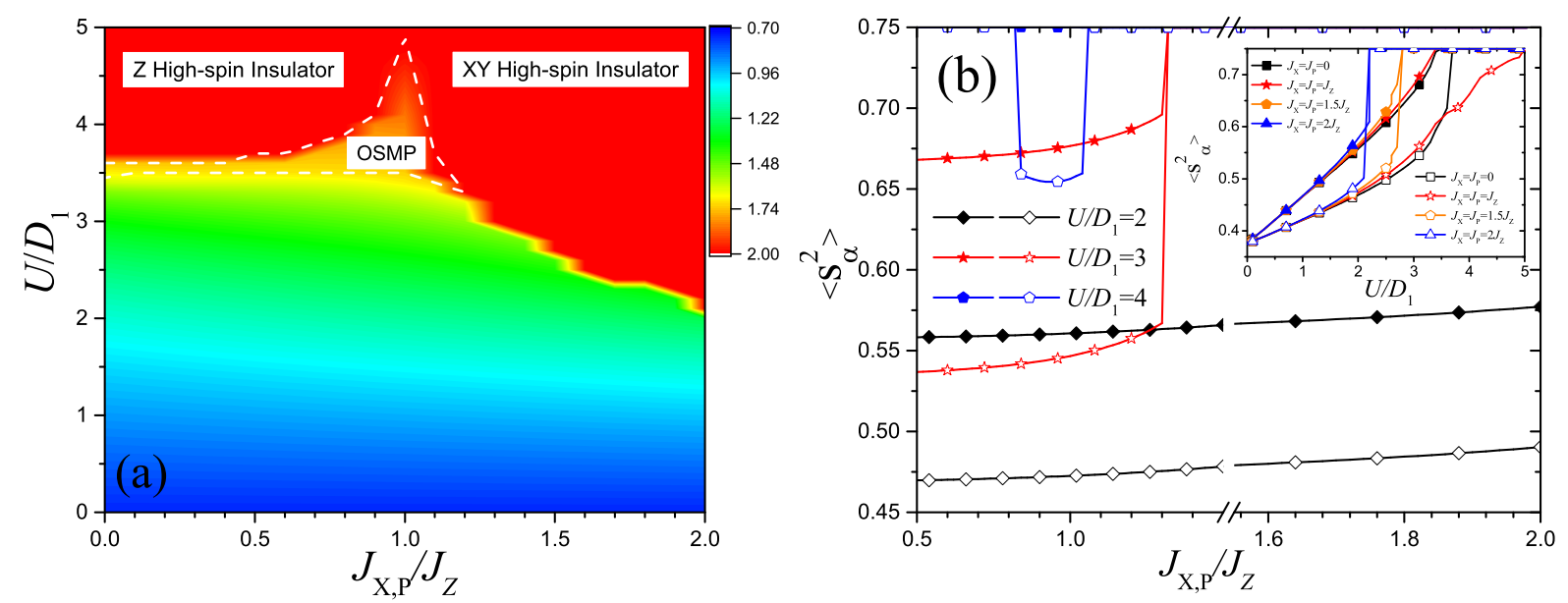

FIG. 5: The moment-square phase diagram of electron correlation $U / D_{1} v s$ anisotropic Hund's rule coupling $J_{X, P} / J_{Z}$ (a) and orbital resolved moment squares for different electron correlations (b) as the functions of transverse Hund's term at $D_{2}=2 D_{1}$ and $n=2$. The insets in (b) show the dependence on $U / D_{1}$ at $J_{X, P} / J_{Z}=0$ (black square), 1 (red star), 1.5 (orange pentagon), and 2 (blue up triangle). The closed and hollowed signs are for orbital-1 and 2, respectively.

around $J_{X, P} / J_{Z} \approx 1$ In this OSMP, the magnetic moment is contributed both by the itinerant and by the local electrons, so that the moment square of total spin is not saturated. In Fig.5(b), the moment squares of spins in two orbitals are plotted for different electron correlations and transverse Hund's terms . Typically, at $U / D_{1}=2$, both magnetic moments slightly increase with lifting $J_{X, P} / J_{Z}$, and total spin is far from saturated; at $U / D_{1}=3$, both spins in two orbitals considerably increase with lifting $J_{X, P} / J_{Z}$, and become fully localized and the total spin is saturated around $J_{X, P} / J_{Z}=1.3$; and at $U / D_{1}=4$, both spins of two orbitals are saturated over whole $J_{X, P} / J_{Z}$, except for the spin of wide band in the OSMP around $J_{X, P} / J_{Z} \approx 1$, as shown in Fig.5(b). In the OSMP region, it is clearly shown that the spin moments of the wide and narrow orbitals become saturated at different correlation strengths, as seen in the inset of Fig.5(b).

To further study the competing roles of longitudinal and transverse Hund's terms, we plot the moment-square-of-spin phase diagram in the $J_{X, P}-J_{Z}$ plane with $U / D_{1}=3$ and $n=2$ in Fig.6. On the whole, there are four or five different phases in the phase diagram, including a small/intermediated-spin correlated metal, a nonmagnetic Mott/orbital insulator, an intermediated-spin OSMP, and high-spin Mott insulator with the spin alignment along the z-axis or in the xy-plane. At $J_{Z}=0$, with increasing $J_{X, P}$, the system undergoes a transition from a cor- 


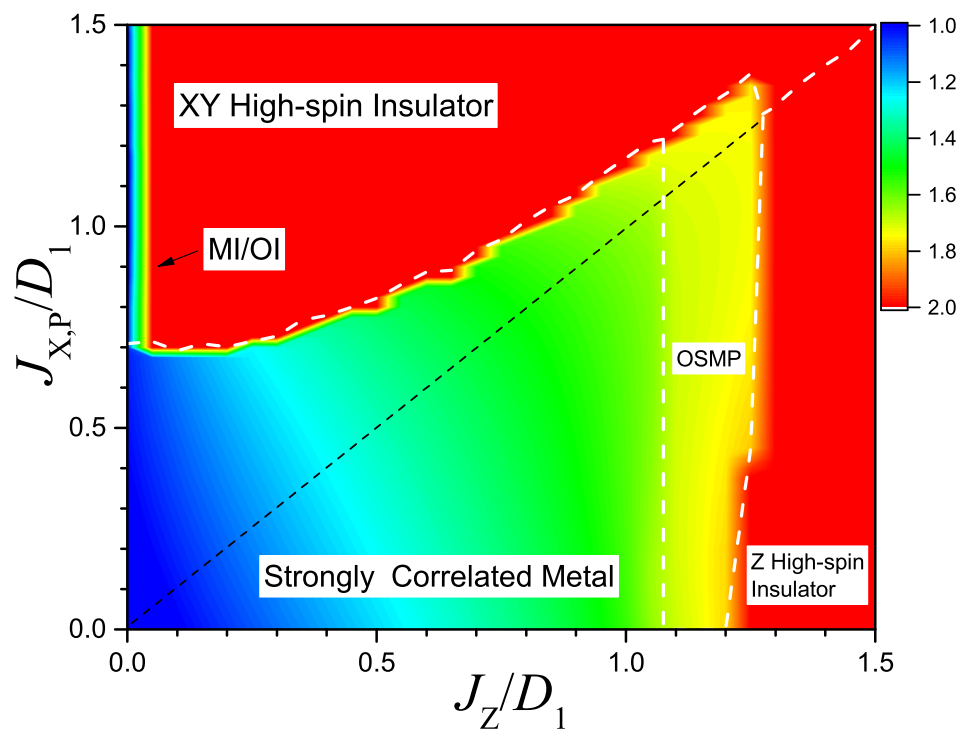

FIG. 6: The moment-square phase diagram of two-orbital Hubbard model in the $J_{X, P}-J_{Z}$ plane. Theoretical parameters are $U / D_{1}=3, D_{2}=2 D_{1}, n=2$ and $\Delta=0$. The contour (color) denotes the value of moment square of spin per site.

related metal with small or intermediate spin to a nonmagnetic Mott/orbital insulator (MI/OI) at the critical $J_{X, P}^{c}$. In the MI/OI phase, the groundstate energies of nonmagnetic Mott insulating and nonmagnetic orbital insulating state are degenerate due to equivalent spin-flip and pair-hopping Hund's terms. The moment square of the spins of the system is rather small in this phase. For finite $J_{Z}$, when $J_{Z} / D_{1}<1.07$ and $J_{X, P} / D_{1}<1$, the system lies in a small-spin or intermediate-spin correlated metallic phase, mainly depending on the magnitude of $J_{Z} / D_{1}$. When $1.07<J_{Z} / D_{1}<1.27$, the narrow orbital becomes insulating due to large electron correlation and the wide one remains metallic, thus the system is an OSMP. As seen in Fig,6, the moment-square of spins in OSMP is obviously larger than that in correlated metallic phase, but remains not saturated.

From Fig.6 we also find that when $J_{Z} / D_{1}>1.25$, the system enters a high-spin Mott insulating phase with dominant $z$-component of spins. In this phase, the interorbital spin correlation functions $<S_{\alpha}^{Z} S_{\beta}^{Z}>$ equals to 0.25 , implying that the spins of two orbitals are parallel and completely localized, and the moment square of spins becomes saturated 2 with $S=1$ and $S^{Z}=1$ or -1 . When $J_{X, P}>J_{Z}$ and above the correlated metallic phase, the system enters to the high-spin Mott insulating phase with dominant xy-component of spins. In this phase, the interorbital spin correlation functions $<S_{\alpha}^{Z} S_{\beta}^{Z}>$ becomes -0.25 , and the moment square of spins becomes saturated 2 , implying that the system has $S=1$ and $S^{Z}=0$. From these results, one sees that the competition 
of transverse Hund's term with the longitudinal one enhances the spin fluctuations, as shown the most broadest intermediated-spin and metallic phase in the $J_{Z}=J_{X}$ line in Fig.6. The direction of local spins depends on the competition between the transverse and longitudinal Hund's terms.

These results demonstrate that the transverse Hund's rule coupling terms are important to the OSMP in two orbital system, and the isotropic Hund's coupling case has strong spin quantum fluctuations. From which, we could understand the small magnetic moments in the ground states of the iron-pnictide superconductors after considering the transverse Hund's coupling terms, since they result in strong spin fluctuations, in agreement with Schickling et al. [46].

\section{Other fractional-filling cases}

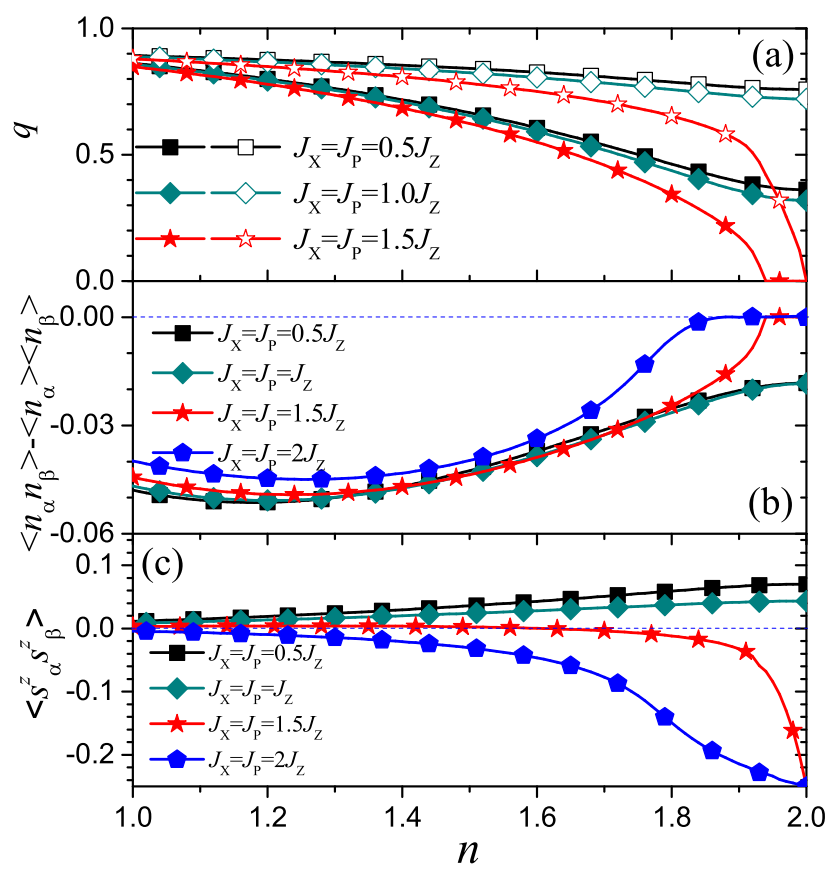

FIG. 7: Quasiparticle weight (a), interorbital charge correlations (b) and z-component correlation functions of interorbital spins (c) as the functions of filling factor for different transverse Hund's rule coupling at $U / D_{1}=3$. The closed and hollowed signitures in (a) are for orbital-1 and 2, respectively.

To further uncover the roles of transverse Hund's terms in other fractional filling cases, we plot the QP weights, interorbital charge and spin correlations as the functions of total filling $n$ for different transverse Hund's coupling terms in the intermediate correlation $U / D_{1}=3$ in Fig. 
7. It shows that the QP weights of the two orbitals show slight decrease with increasing $n$ when $J_{X, P} / J_{Z}<1$ or $J_{X, P} / J_{Z}=1$; at $J_{X, P}=1.5 J_{Z}$, the QP weights first gradually decrease, then sharply drop to zero subsequently when the total particle number is closing to half filling of $n=2$, as shown in Fig. 7(a). The system undergoes from metal to OSMP phase in the vicinity of half filling, where the narrow orbital becomes insulating with the wide one remaining metallic; its mechanism is similar to that at half filling, i.e., large transverse Hund's terms enhance the effective electron correlation of the system. So the narrow orbital becomes half filled and insulating, and the wide one remains metallic at the same time. At $n=2$, the system enters to the insulating phase.

In $1.4<n<1.8$, the transverse Hund's rule coupling terms slightly enhance the interorbital charge correlation, as seen in Fig. 7(b). This arises from the pair hopping term since the pair occupation is still considerable. Further, in the OSMP region, the narrow orbital becomes half filled and insulating, thus the interorbital charge correlation becomes zero. The interorbital spin correlations as the functions of filling factor in Fig. 7(c) show that the spins of the electrons in two orbitals tend to be parallel when $J_{X, P} / J_{Z}<1.5$ and be antiparallel when $J_{X, P} / J_{Z}=1.5$; meanwhile, the electron localizations in the two orbitals are enhanced with increasing filling factor $n$.

On the other hand, in strong correlation regime of $U / D_{1}=10$, the filling-factor dependence of the QP weights, interorbital charge and spin correlations on transverse Hund's coupling are significantly different, as plotted in Fig. 8. It is seen in Fig. 8(a) that in strong correlation case, the bandwidths of two orbitals are seriously renormalized by the transverse Hund's terms. The QP weights of the narrow orbitals for various fillings first fall into zero, while these of the wide ones in are considerably different. The QP weight of the isotropic case is the largest since its spin fluctuation and itinerancy of the electrons are enhanced by the spin flip term. When $n>1.7$, the narrow orbitals of all filling factors become insulating, the wide ones remain meatllic. The system transits to the OSMP and the area of OSMP shrinks considerably with increasing $J_{X}$ and $J_{P}$ when $J_{X, P} / J_{Z}>1$, as seen in Fig. 8(a). This arises from the fact that the electrons tend to distribute in different orbitals, prefer to fractionally occupy these two orbitals in the systems with large transverse Hund's terms. Thus the OSMP is suppressed by the spin-flip terms in the systems away from half filling.

Fig. 8(b) shows that the spin-flip Hund's term suppresses the interorbital charge correlation. However, the sign of the interorbital charge correlation becomes positive with $J_{X, P} / J_{Z}=2$ in the region of $1.3<n<1.9$, which is mainly due to the spin flip process enhanced by the spin-flipping Hund's term. The interorbital spin correlations in Fig. 8(c) also show that the z-component spins 


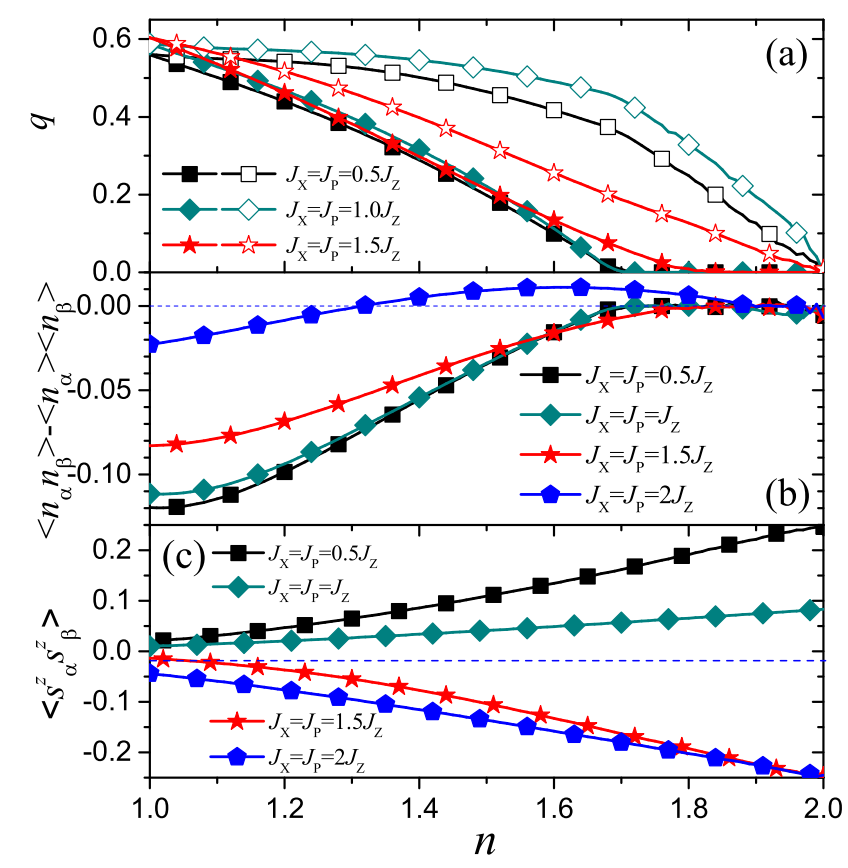

FIG. 8: Quasiparticle weight (a), interorbital charge correlations (b), and z-component correlation functions of interorbital spins (c) as the functions of filling factor for different transverse Hund's rule coupling at $U / D_{1}=10$. The closed and hollowed signitures in (a) are for orbital-1 and 2, respectively.

of the electrons in two orbitals tend to antiparallel when $J_{X, P} / J_{Z}>1$; on the other hand, the Z-component spins parallel when $J_{X, P} / J_{Z}<1$. Again, these results in Fig. 7(c) and Fig. 8(c) demonstrate that a magnetic quantum fluctuation region along the $J_{X, P}=J_{Z}$.

\section{DISCUSSIONS AND CONCLUSIONS}

We have presented the effects of spin-flip and pair-hopping Hund's terms on MIT and OSMP by using rotationally invariant slave boson approach. As a competitor of the longitudinal Hund's term, the spin-flip and pair-hopping Hund's terms may display two important influences on MIT and OSMP: the first one is that they may suppress the interorbital charge correlations and distribute the electrons in different orbitals; the second one is that they may enhance the intraorbtial spin fluctuations, especially around the half filling. These two influences enhance the electron itineracy, and is not favor of the MIT when $J_{X, P}<J_{Z}$. Away from quarter filling, the OSMP is favorable by the spin-flip and pair-hopping terms in the strong correlation regime. When $J_{X, P}>J_{Z}$, we find that 
the spin-flip terms enhance the itinerancy of electrons in the quarter filling case, since the orbital pair-hopping is negligible and the intraorbital charge and spin fluctuations are not sensitive to the spin-flip and pair-hopping Hund's terms; however, in the half filing case, the effective electron correlations of the system are strongly enhanced by the transverse Hund's terms, and the OSMP region gradually reduces and even disappears.

(a)
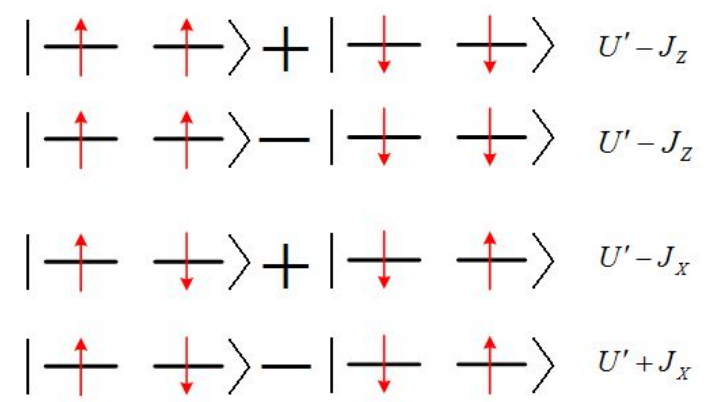

(c)

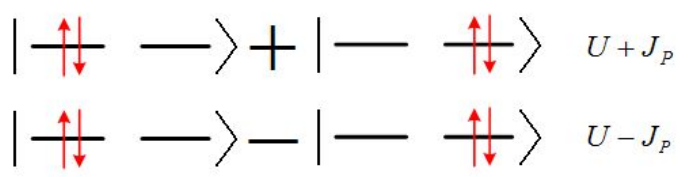

FIG. 9: The double-occupied atomic configurations of the spin-parallel Fock state (a), spin-antiparallel Fock state (b) and low-spin orbital Fock state (c), which are favored by $J_{Z}, J_{X}$ and $J_{P}$, respectively, in two-orbital systems. The corresponding eigen energy of each Fock state is also given in the right.

From the double occupied Fock states shown in Fig.9 one could clearly explain the different effects of the longitudinal $J_{Z}$ and transverse (spin-flip $J_{X}$ and pair-hopping $J_{P}$ ) Hund's couplings, i.e., $J_{Z}$ favors the spin-parallel Fock state (a) and $J_{X}$ or $J_{P}$ favors the system in the spin-antiparallel Fock state (b) or low-spin orbital Fock state (c), respectively. Therefore one could understand the competing roles of the longitudinal and transverse Hund's coupling terms, since they favor different spin and orbital states.

From our study we have shown that the present asymmetric two-orbital system with finite correlation has an particular parameter region around $J_{X, P} \approx J_{Z}$, along which the system has most strong spin fluctuations, and it approximately separates the parametric space $J_{X, P} / J_{Z}$ into two parts: (I) the interorbital spin correlation function in the z-direction is positive and the interorbital spins are parallel in the $J_{X, P} / J_{Z}<1$ region, while the interorbital spins are antiparallel in the $J_{X, P} / J_{Z}>1$ region; (II) in the $J_{X, P} / J_{Z}<1$ region the transverse Hunds terms enhance spin fluctuations and favor the itineracy of electron, while they enhance the electronic correlations in the $J_{X, P} / J_{Z}>1$ 
region, hence favor the occurrence of the MIT; and correspondingly, (III) the OSMP is favorable in the $J_{X, P} / J_{Z}<1$ region, while it is unfavorable in the $J_{X, P} / J_{Z}>1$ region.

In summary, we have shown that the competing role of the longitudinal and transverse Hund's coupling terms on metal-insulator transition and orbital selective Mott phase in two-orbital halffilled Hubbard model arises from the spin-antiparallel double-occupied Fock state favored by the spin-flip term and the low-spin double occupied Fock state by the pair-hopping term. According to the present results we are also able to understand the small magnetic moment in iron-pnictide superconductors. These results demonstrate that the transverse Hund's terms becomes an important factor both in the Hund's metal materials and the systems in the brink of Mott insulating phase and orbital selective Mott phase.

\section{Acknowledgments}

This work was supported by the National Science Foundation of China under Grant no. 11204311, 11274310 and 11104274. Numerical calculations were performed at the Center for Computational Science of CASHIPS.

\section{APPENDIX A: SUPPLEMENTAL MATERIAL: COMPARISON BETWEEN THE SLAVE BO- SON AND DYNAMICAL MEAN FIELD THEORY RESULTS}

We present the numerical results on the paramagnetic metal-insulator transitions, the quasiparticle weight, and inter-orbital charge correlation functions in two-orbital Hubbard models obtained by our Slave Boson method, and compare our data with those obtained by the DMFT method.

In the present asymmetric two-orbital situation, the results obtained by the Slave Boson method in Fig.10 (a) and by the DMFT method in Fig.10(b) [4] show that the metal-insulator transition occurs almost at the same critical correlation strength; and at $J_{X, P}=J_{Z}=0$, the paramagnetic orbital selective Mott phase is not observed, in agreement with the results obtained by the DMFT+QMC method in Fig.10(b). The effect of Hund's rule coupling on metal-insulator transition is shown in Fig.11. In comparing the slave boson and DMFT results shown in Fig.11, we find that the metalinsulator transition occurs at $U / D_{1}=2.2$ when $J_{X, P}=J_{Z}=0.25 U$ (see Fig.11(a)), which is in consistent with the DMFT results as shown in Fig.11(b). With decreasing Hund's coupling terms, the critical electron correlation strength increases. The inter-orbital charge correlation functions 

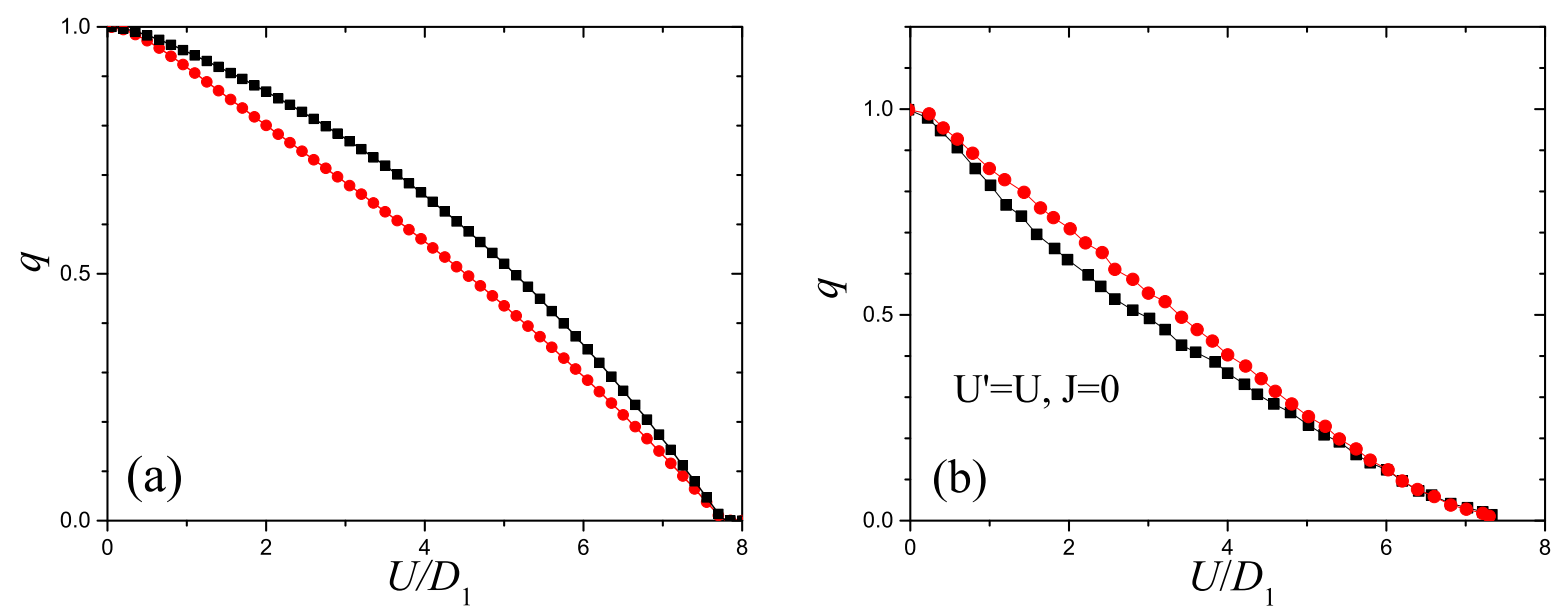

FIG. 10: Dependence of the quasi-particle weights on Coulomb correlation in two-orbital Hubbard model. Theoretical parameters are $D_{2}=2 D_{1}, J_{X, P}=J_{Z}=0, n=2$. Left: our results(a), Right: DMFT data(b)[4]
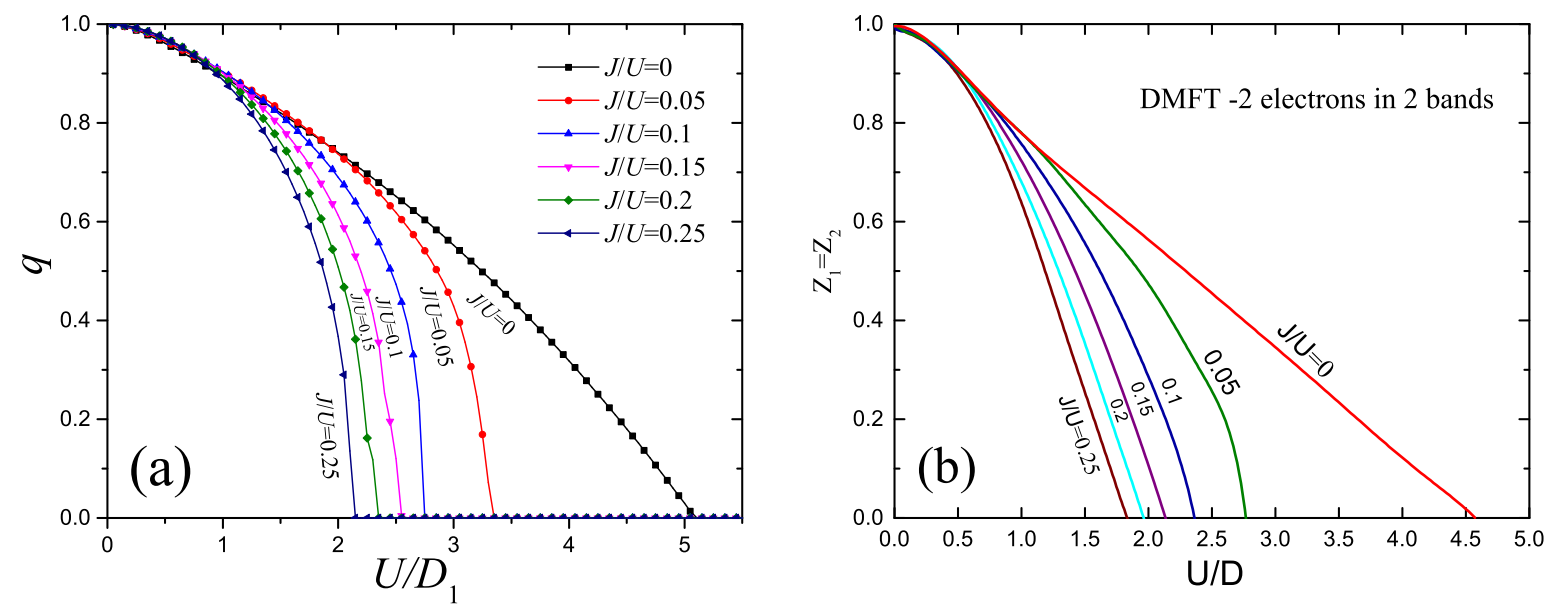

FIG. 11: Comparison of the dependence of quasi-particle weights on the electronic correlation $U$ and the Hund's rule coupling strength obtained by the slave boson method(a) and the DMFT method(b) [10] given by Slave Boson and DMFT methods are in agreement very well, as shown in Fig. 12 (a) and (b) respectively. This also demonstrates that the inter-orbital charge correlation can also be well described by slave boson method. Notice that the transverse-axes of these two figures are plotted 

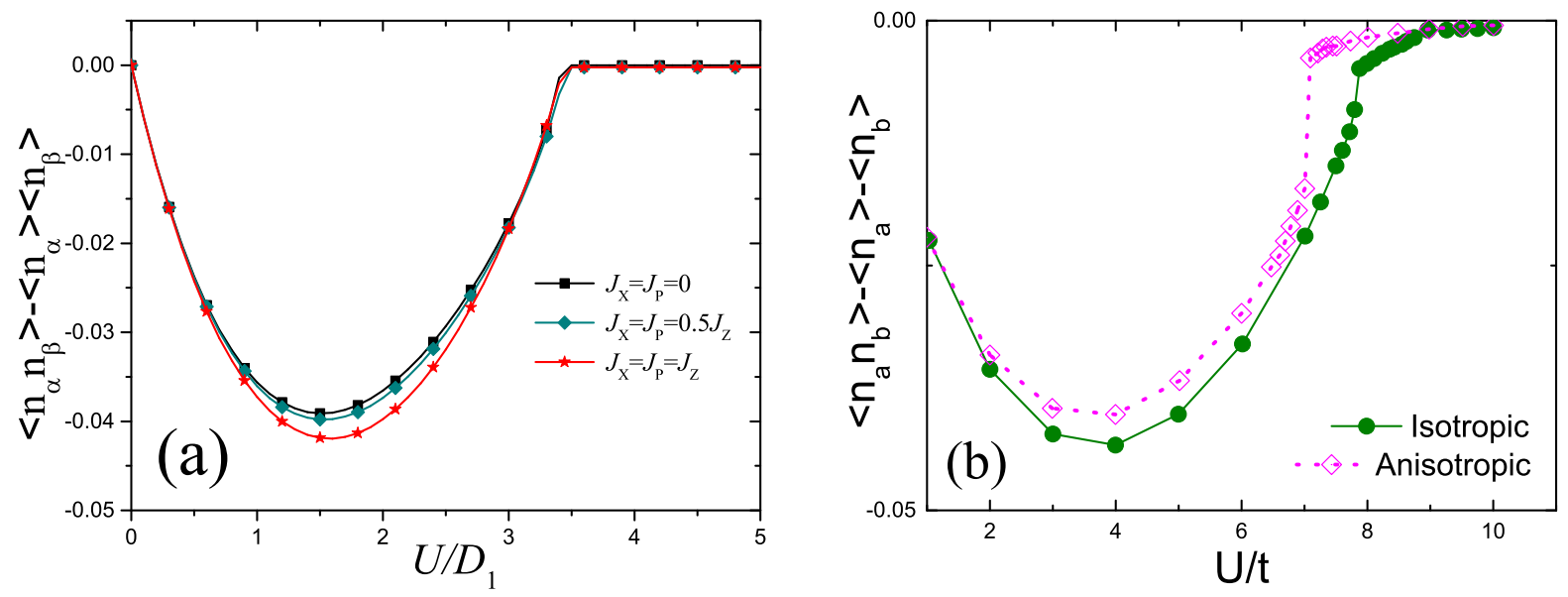

FIG. 12: Comparison of the inter-orbital charge correlations obtained by the Slave Boson method(a) and by the DMFT theory(b) [47]

in $U / D_{1}$ and in $U / t$, respectively.

[1] V. I. Anisimov, I. A. Nekrasov, D. E. Kondakov, T. M. Rice and M. Sigrist, Eur. Phys. J. B. 25, 191 (2002).

[2] L. Balicas, S. Nakatsuji, D. Hall, T. Ohnishi, Z. Fisk, Y. Maeno and D. J. Singh, Phys. Rev. Lett. 95, 196407 (2005).

[3] A. Shimoyamada, K. Ishizaka, S. Tsuda, S. Nakatsuji, Y. Maeno and S. Shin, Phys. Rev. Lett. 102, 086401 (2009).

[4] A. Koga, N. Kawakami, T. M. Rice and M. Sigrist, Phys. Rev. Lett. 102, 086401 (2009).

[5] L. Huang, L. Du and X. Dai, Phys. Rev. B 86, 035150 (2012).

[6] T. Kita, T. Ohashi and N. Kawakami, Phys. Rev. B 84, 195130 (2011).

[7] L. de' Medici, J. Mravlje and A. Georges, Phys. Rev. Lett. 107, 256401 (2011).

[8] A. Rüegg, M. Indergand, S. Pilgram and M. Sigrist, Eur. Phys. J. B 48, 55 (2005).

[9] L. de' Medici, S. R. Hassan, M. Capone and X. Dai, Phys. Rev. Lett. 102, 126401 (2009).

[10] L. de' Medici, Phys. Rev. B 83, 205112 (2011).

[11] P. Werner, E. Gull and A. J. Millis, Phys. Rev. B 79, 115119 (2009).

[12] F. Han, X.G. Wan, B. Shen, and H. H. Wen, Phys. Rev. B 86, 014411 (2012).

[13] Z. Wang, M. Schmidt, J. Fischer, V. Tsurkan, M. Greger, D. Vollhardt, A. Loidl and J. Deisenhofer, 
Nature Communications. 5, 3202 (2014).

[14] W. Li, C.F. Zhang, S.H. Liu, X.X. Ding, X.W. Wu, X.Y. Wang, H. H. Wen, and M. Xiao, Phys. Rev. B 89, 134515 (2014).

[15] D. Ootsuki, N. L. Saini, F. Du, Y. Hirata, K. Ohgushi, Y. Ueda, and T. Mizokawa, Phys. Rev. B 91, 014505 (2015).

[16] M. Yi, D. H. Lu, R. Yu, S. C. Riggs, J.-H. Chu, B. Lv, Z. K. Liu, M. Lu, Y.-T. Cui, M. Hashimoto, S.-K. Mo, Z. Hussain, C. W. Chu, I. R. Fisher, Q. Si and Z.-X. Shen, Phys. Rev. Lett. 110, 067003 (2013).

[17] F. Hardy, A. E. Böhmer, D. Aoki, P. Burger, T. Wolf, P. Schweiss, R. Heid, Phys. Rev. Lett. 111, $027002(2013)$.

[18] L. Craco, M. S. Laad and S. Leoni, J. Phys.: Condens. Matter 26, 145602 (2014).

[19] Y. Z. You, F. Yang, S. P. Kou and Z. Y. Weng, Phys. Rev. Lett. 107, 167001 (2011).

[20] N. Lanatà, H. U. R. Strand, G. Giovannetti, B. Hellsing, Luca de' Medici and M. Capone, Phys. Rev. B 87, 045122 (2013).

[21] R. Yu and Q. M. Si, Phys. Rev. Lett. 110, 146402 (2013).

[22] R. Yu and Q.M. Si, Phys. Rev. B 84, 235115 (2011).

[23] G. Giovannetti, Luca de' Medici, M. Aichhorn, and M. Capone, Phys. Rev. B 91, 085124 (2015).

[24] L. de' Medici, G. Giovannetti and M. Capone, Phys. Rev. Lett. 112, 177001 (2014).

[25] D. Y. Liu, Y. M. Quan, X. J. Zheng, X. L. Yu and L. J. Zou, J. Phys.: Condens. Matter 25, 125601 (2013).

[26] P. Werner and A. J. Millis, Phys. Rev. Lett. 95, 126405 (2007).

[27] A. Libsch, Phys. Rev. Lett. 95, 116402 (2005).

[28] S. Biermann, L. de' Medici and A. Georges, Phys. Rev. Lett. 95, 206401 (2005).

[29] Z. P. Yin, K. Haule, and G. Kotliar, Nat. Mater. 10, 932 (2011).

[30] L. Fanfarillo, E. Bascones, arXiv. 1501.04607v2 (2015).

[31] C. Aron, and G. Kotliar, Phys. Rev. B 91, 041110(R) (2015).

[32] Y. M. Quan, L. J. Zou D. Y. Liu and H. Q. Lin, Eur. Phys. J. B 85, 55 (2012).

[33] L. de' Medici, A. Georges and S. Biermann Phys. Rev. B 72, 205124 (2005).

[34] E. Jakobi, N. Blümer and P. V. Dongen, Phys. Rev. B 87, 205135 (2013).

[35] E. Jakobi, N. Blümer and P. V. Dongen, Phys. Rev. B 80, 115109 (2009).

[36] F. Lechermann, A. Georges, G. Kotliar and O. Parcollet, Phys. Rev. B 76, 155102 (2007). 
[37] R. Frésard and G. Kotliar, Phys. Rev. B 56, 12909 (1997).

[38] J. Kotliar and A. E. Ruckenstein, Phys. Rev. Lett. 57, 1362 (1986).

[39] T. J. Li, Y. M. Quan, D. Y. Liu and L. J. Zou, J Magn Magn Mater 324, 1046 (2012).

[40] H. Hasegawa, Phys. Rev. B 56, 1196 (1997).

[41] R. Frésard and P. Wölfle, Int. J. Mod. Phys. B 6, 685 (1992).

[42] T. Li, P. Wölfle and P. J. Hirschfeld, Phys. Rev. B 40, 6817 (1989).

[43] Y. M. Quan, L. J. Zou and D. Y. Liu, Acta Phys.Sin 61, 017016 (2012)[in Chinese].

[44] Y. M. Quan, Q. W. Wang, D. Y. Liu, X. L. Yu, L. J. Zou, Computer Physics Communications 191, 90-99 (2015).

[45] L. de' Medici, S. R. Hassan and M. Capone, J Supercond Nov Magn 22, 535-538 (2009).

[46] T. Schickling, F. Gebhard, and J. Bünemann, Phys. Rev. Lett. 106, 146402 (2011).

[47] Z. Y. Song, H. Lee and Y. Z. Zhang, New J. Phys 17, 033034 (2015). 